Aus der Universitäts-Frauenklinik in Strassburg i. E.

\title{
Zur mikroskopischen Diagnose der abgelaufenen Schwangerschaft.
}

Von

\author{
Privatdozent Dr. G. Schickele.
}

(Hierzu Tafel IV und 4 Textfiguren.)

Die sichere Erkennung einer abgelaufenen Schwangerschaft bei klinisch unbestimmten Merkmalen ist immer noch ein Stiefkind der mikroskopischen Untersuchung. Vor Jahren hat sich 0 pitz $^{1}$ ) bemüht, die Erkennung dieser Fälle zu fördern. Er hat neben den Drüsenveränderungen, welche er als charakteristisch ansah, die aber den Einwänden von Seitz ${ }^{2}$ ) und erst recht den heutigen Kenntnissen über die prämenstruelle Umwandlung der Schleimhaut nicht mehr Stand halten können, alle Merkmale aufgezählt, welche zur Erkennung einer abgelaufenen Schwangerschaft beitragen können. So wertvoll diese Zusammenstellung auch ist, müssen wir trotzdem bis heute immer noch den Nachweis von Chorionzotten zur Sicherstellung der Diagnose verlangen. In zweiter Linie kommt der Decidua in zusammenhängenden Verbänden eine wesentliche Bedeutung bei. Sie wird aber dadurch abgeschwächt, dass unveränderte Deciduabezirke einige Wochen nach einer abgelaufenen Schwangerschaft meist nicht mehr vorkommen, es sei denn, dass auch Zotten zurückgeblieben sind. Bei länger retinierten Resten leidet die Decidua häufig zu allererst, so dass auch sie dann kein sicheres Merkmal mehr abgeben kann. Wenn es sich nur um einige Deciduazellen handelt, dann muss man sehr vorsichtig sein. Da deciduaähnliche Zellen auch bei der Menstruation vorkommen, können sie zur Erkennung einer Schwangerschaft nicht in ein-

1) Zeitschr. f. Geburtsh. u. Gyn. 1900.

2) Zeitschr. f. Geburtsh. u. Gyn. 1901.

Archiv für Gynäkologie, Bd. 94. H. 1. 
82 Schickele, Mikroskopische Diagnose der abgelaufenen Schwangerschaft.

deutiger Weise verwerthet werden. Die Chorionzotten, insbesondere ihre Ektodermzellen, sind sehr widerstandsfähig; diese werden noch zu einer Zeit gefunden und als solche erkannt, wo die übrigen Bestandteile der Zotten in vorgeschrittener Degeneration sich befinden. Fehlen aber die Ektodermzellen, dann sind lange zurückgebliebene Chorionzotten häufig nicht mehr mit Bestimmtheit zu identifizieren, so dass auch sie zur Sicherung der Diagnose kaum noch beitragen können. Alle anderen Befunde (vergl. Opitz, 1. c. S. 36, II., 1-6) haben für sich allein, ohne Zotten und Decidua keine Beweiskraft.

Im Verlaufe gleichzeitiger Untersuchungen über Gefässveränderungen an der Placentarstelle und über ausgeschabte Schleimhautbröckel ist mir ein Merkmal aufgefallen, welches zur Erkennung abgelaufener Schwangerschaften beitragen dürfte. Vor Kurzem konnte ich nachweisen, dass schon in den ersten Monaten der Gravidität die Arterien der Placentarstelle bestimmte Umwandlungen erleiden ${ }^{1}$ ). Die Gefässwände werden hyalin, und zwar entwickelt sich dieser Vorgang von aussen nach innen. Hieran sind wohl die durch die Schwangerschaft an sich gegebenen Zirkulationsveränderungen schuld (Hyperämie), weit mehr aber die heterolytische Wirkung der in den Gefässwänden liegenden fötalen Zellen. Schon rom Ende des 2. Schwangerschaftmonats ab kann man innerhalb der hyalinen Arterienwände Ektodermzellen nachweisen. Dieses Merkmal bleibt bis zum Ende der Schwangerschaft bestehen, mit der gelegentlichen Modifikation, dass in den letzten Monaten die Zahl dieser Zellen abnehmen kann. Nach dieser Feststellung kann es nicht mehr merkwürdig erscheinen, wenn z. B. in dem wegen Blutungen nach unvollständigem Abort ausgeschabten Uterusinhalt hyaline Gefässe mit Ektodermzellen neben Chorionzotten u. s. w. gefunden werden. Wir wissen ferner, dass Ektodermzellen in retinirten Eitheilen besonders widerstandsfähig sind, deshalb noch gut erhalten und deutlich erkennbar sein können zu einer Zeit, wo die anderen Eireste schon degeneriert sind. Dies gilt auch für die in den hyalinen Gefässwänden liegenden Ektodermzellen. Diese Zustände können in verschieden lange Zeit retinierten Eiresten in den entsprechenden Abstufungen ohne Schwierigkeit verfolgt werden.

Zur Orientierung einige Fälle:

1) Vergl. Beiträge z, Geburtsh, u. Gyn. 1911. 
1. Ausschabung No. 226. 39 Jahre alte Frau. 8 Entbindungen. Letzte Regel 24. Juni. Am 30. VIII. ganz geringer Blutabgang, der sich nach 14 Tagen wiederholt. Dabei starke Schmerzen im Leib und in der linken Seite. Vor 3 Wochen erneute Blutung, die mit geringen Unterbrechungen bis zum Eintritt in die Klinik anhält. Die Pat. ist vom Arzt geschickt wegen Verdachts auf Extrauteringravidität oder Abort. Befund: Vulva etwas livid, äusserer Muttermund geschlossen, Uterus leicht retroflektiert, kleinfaustgross, beweglich. Linkes Ovarium tiefstehend, kleinfaustgross, fixirt. Ausschabung am 23. X. 1909: Viele Chorionzotten, in beginnender Degeneration, einige schon intensiv verändert, ihre Ektodermzellen aber im Allgemeinen noch deutlich; Decidnazellen gut erhalten; zwischen ihnen zahlreiche Gefässe mit gleichmässig hyaliner Wand, in der typische Ektodermzellen liegen. Die Schleimhautdrüsen sind klein, ihre Grenzen scharf; Epithelien gut gefärbt; das Zwischengewebe zellreich; nirgends erweiterte oder geschlängelte Drüsen.

2. Ausschabung 212. 31jährige Frau. 4 Geburten. Letzte Regel im Juni. Blutung seit 12 Wochen. Befund: Aeusserer Muttermund geschlossen. Uterus vorn, kleinfaustgross, höckerig. Ausschabung am 29. IX. 1909: Zahlreiche Chorionzotten, ziemlich gut erhalten, mit Ektodermbesatz. Viel Fibrin in kleinen Schollen und in grossen Haufen. Nekrotische Bezirke zum Theil aus feingekörntem Material bestehend. Kleine hyaline Stellen in der Musculatur, ebenso um die äussere Schicht hier liegender Gefässe. Andere Gefässe mit vollständig hyaliner Wand, in der schön erhaltene Ektodermzellen liegen. Dieselben Zellen sind ansserdem in der Muscularis und Mucosa gut erhalten. Die Drüsen mit scharfen Umrissen, eng, nicht geschlängelt, tragen gut gefärbtes Epithel. Das Zwischengewebe ist zellreich (Leukozyten). Keine Deciduazellen.

3. Ausschabung No. 249. 27jährige Frau. 11 Schwangerschaften, darunter mehrere Aborte; vor 7 Wochen Fehlgeburt im 3. Monat. Seither nnregelmässige Blutungen, seit 5 Wochen angeblich ununterbrochen. Befund: Uterus nicht wesentlich vergrössert; äusserer Muttermund geschlossen, Ausschabung am 4. XII. 1909: Es fallen zahlreiche kleine hyaline Zellen oder Flecken innerhalb der Schleimhaut und Muscularis von vornherein auf. Die äussere Schicht mancher Gefässe ist hyalin verändert. Die Wand anderer Gefässe ist in ihrer ganzen Breite hyalin; in ihr einige deutliche Ektodermzellen, die selbst in ganz kleinen Gefässen dureh ihre Grösse und intensive Färbung auffallen (Tafel IV, Fig. 1). Das Endothel der grösseren Gefässe ist erhalten. In dem Lumen liegen rote Blutkörperchen. Andere kleine Gefässe sind obliterirt und erscheinen als helle hyaline Kreise, in denen noch 2-4 Ektodermzellen liegen (vergl. Tafel IV, Fig. 1 rechts oben). Reste des Lumens sind manchmal noch angedentet. Chorionzotten sind fast homogen und hyalin, als solche kaum noch zu erkennen. Manche unter ihnen sind jedoch durch ihren nicht vollständig untergegangenen Ektodermbesatz noch einwandsfrei festzustellen. Die in den genannten hyalinen Gefässen liegenden Ektodermzellen sind wesentlich besser erhalten als jene der Zottenreste. Die Drüsen sind wenig geschlängelt, mässig secernierend. Epithelien meist hoch, gut gefärbt; daneben auch ganz kleine enge Drüsen. Das Zwischengewebe zellreich, Deciduazellen fehlen.

4. Ausschabung No. 206. 32jährige Fünftgebärende. Periode angeblich nicht ausgeblieben. Letzte Periode am 10. XI.; 7 Tage nach deren Aufhören plötzlich starke Blutung, angeblich mit Ohnmachtsanfall. 
Befund: Utertus nicht wesentlich vergrössert, in Anteflexio, Os ext. klafft. Am 19. XI. Ausschabung: Zahlreiche kleine Drüsen; Gefässe mit schöner, breiter, hyaliner Wand, in der Ektodermzellen meist in grosser Zahl liegen. Das Endothel ist nicht immer deutlich. In den Lumina meistens rote Blutkörperchen (Tafel IV., Fig. 2, 3, Textfig. 1). Deciduazellen sind spärlich vorhanden. Einzelne rundliche weisse Gebilde sind mit Rücksicht auf ihren theilweise erhaltenen Ektodermbelag wohl als alte Chorionzotten aufzufassen. Sie treten den eben beschriebenen Gefässen gegenüber vollständig in den Hintergrund. Die Drüsen scharf gezeichnet, zum Teil geschlängelt und erweitert, ihre Epithelien deutlich, ziemlich hoch.

\section{Figur 1. (Aus der Ausschabung 206.)}

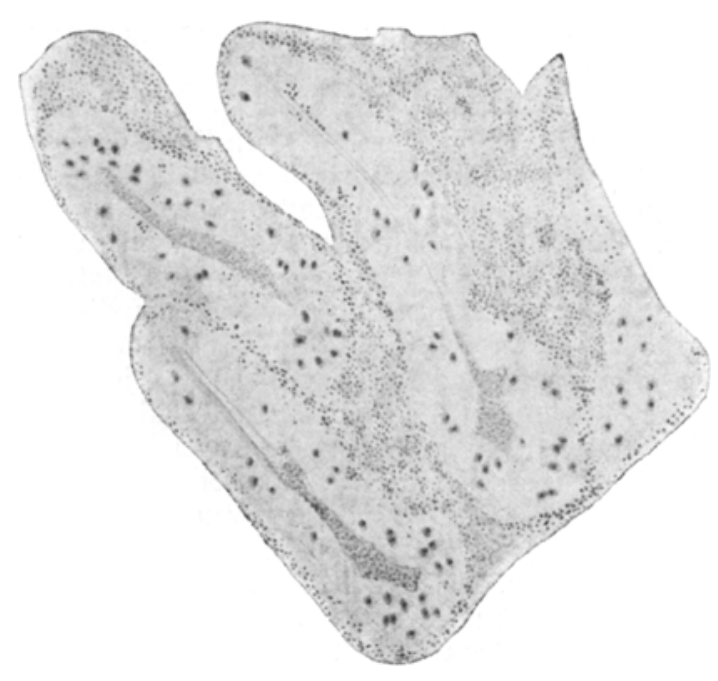

Wohrere mittelgrosse Gefässe mit breiter hyaliner Wand, in der Ektodermzellen liegen. (Die Zellen sind in Wirklichkeit grösser als sie in der Figur wiedergegeben sind.) Das Endothel ist nicht gleichmässig gut erhalten. In diesen Gefässen liegt frisches Blut.

5. Ausschabung No. 342. 35 jährige Mehrgebärende. Letzte Regel 15. bis 23. III. 1910; im April ausgeblieben. Vom 7. Mai an 14 Tage lang ununterbrochen. Nach 8tägiger Pause erneute Blutung bis kurz vor der Aufnahme. Befund: Linke Unterleibsseite druckempfindlich. Uterus in beweglicher Retroflexio, etwas vergrössert. Ausschabung am 28. VI.: Zahlreiche sekretreiche Drüsen, sägeförmig, stellenweise mit büschelförmigen Vorsprüngen in das Lumen. Die Epithelien scharf gezeichnet, mässig hoch, Zwischengewebe zellreich. Bei der Durchmusterung der zuerst angefertigten Schnitte fallen sofort mehrere Gefässe mit breiter hyaliner Wand anf, in der deutliche Ektodermzellen liegen (Textfig. 2). Das Endothel ist vielfach noch gut erhalten. In den engen Lumina zuweilen noch Blut. Nach diesem Befund wurde die Diagnose auf einen voraufgegangenen Abort gestellt und nun von dem gesammten Material der Ausschabung zahlreiche Schnitte angefertigt und auf weitere Schwangerschaftsbeweise gefahndet. In zahlreichen Schnitten fand sich in- 
mitten von hyalinen Massen ein Arterienknäuel (Textfig. 3), dessen einzelne Bestandteile schon ziemlich weit degeneriert waren. An manchen Gefässwänden scheint eine bindegewebige Wucherung vorhanden zu sein. mit nachfolgender Verengerung des Lumens. Innerhalb des Fibrinbezirkes liegen mehrere blassgefärbte grosse Zellen (Ektodermzellen). Die Gefässwände selbst nehmen nur noch eine diffuse Färbung an. In ihnen Reste von Zellen. In vielen Lumina rote Blutkörperchen. Ausserdem wurden einige ziemlich degenerirte Chorionzotten mit theilweise erhaltenem Ektodermbesatz gefunden. Deciduazellen fehlen.

6. Ausschabung No. 284. 29 jährige Pat. 5 Geburten, die letzte vor 11 Monaten. Seit dieser Geburt tritt die Regel fast alle 14 Tage auf, 8-10 Tage dauernd. Befund: Uterus etwas vergrössert. Aeusserer

Figur 2. (Ausschabung 342.)

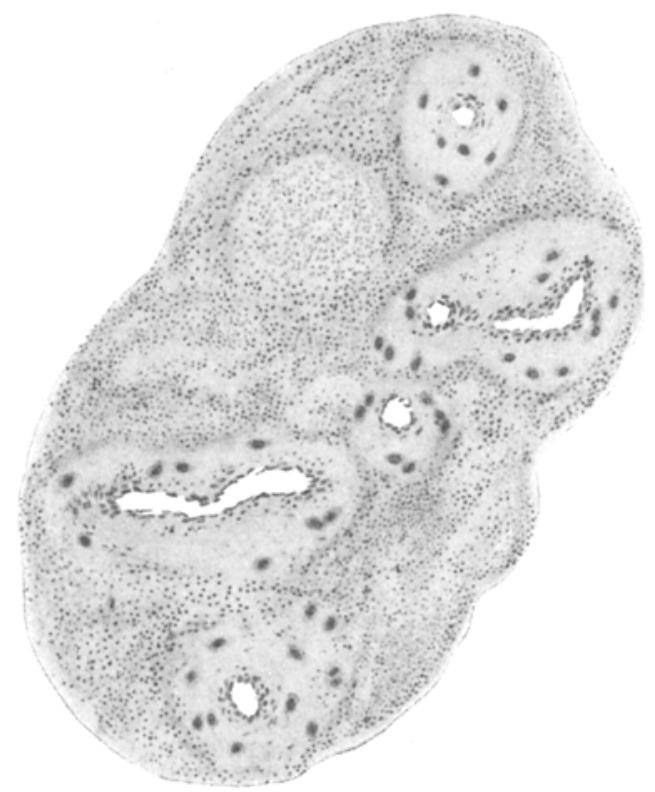

Inmitten zellreichen Stromagewebes liegen mehrere Gefässe mit gut erhaltenem Fndothel and breiter, stellenweise verdickter, hyaliner Wand; in ihr deutliche Ektodermzellen. Dem Endothel benachbart - übriggebliebene oder neugebildete? - Bindegewebszellen.

Muttermund geschlossen. Ausschabung am 3. III. 1910: Ziemlich reichliches Material, mit vielen Fetzen Muscularis. In der Schleimhaut und Muscularis zahlreiche dickwandige Gefässe, deren äussere Schicht gequollen und hyalin ist; ausser dem Gefässe mit ganz hyaliner Wand, in der gut erhaltene Ektodermzellen liegen, entsprechend den Figuren 1. und 2. Das Endothel ist in. manchen Gefässen vollständig intakt. In den Lumina Blut. Manche der genannten Gefässe sind obliteriert, unterscheiden sich aber sonst nicht ron den. vorigen. Chorionzotten und Deciduazellen sind nicht gefunden worden. Die Drüsen eng, klein, weit 
86 Schickele, Mikroskopische Diagnose der abgelaufenen Schwangerschaft.

auseinander stehend; nur einige leicht geschachtelte Drüsen liegen ziemlich dicht. Zwischengewebe mässig zellreich.

Diese Fälle sind ausgewählt unter einem grossen Material. Klinisch sichere Fälle von vorhergegangener Gravidität sind absichtlich weggelassen, da es sich hier nur um solche handeln soll, die anamnestisch unsicher, trotz mikroskopischer Untersuchung der Ausschabung von vornherein nicht immer sichergestellt werden konnten. Sie dürften immerhin genügen, um die folgenden Ausführungen zu belegen.

Figur 3. (Ausschabung 342.)

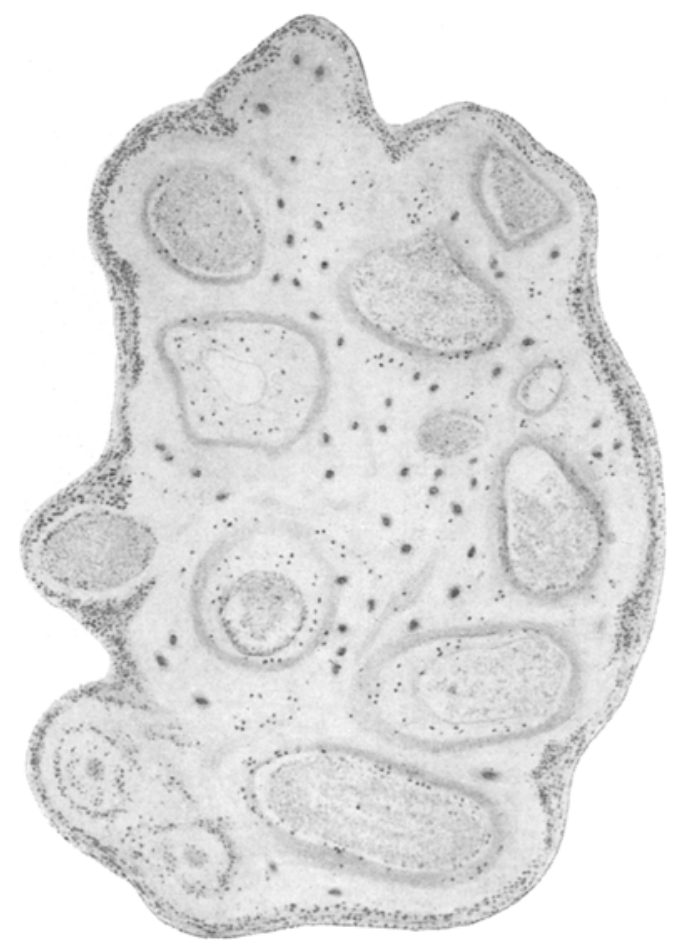

Arterienknäuel, inmitten eines grösseren zusammenhängenden Fibrinbezirkes, in dem Ektodermzellen liegen.

(Vergl. Langhans, Beitr. zur Geburtsh. u. Gyn. Bd. V.)

In vielen Fällen deutet die Anamnese nicht mit Sicherheit auf Gravidität, so dass man nach ihr allein schwanken könnte zwischen der Möglichkeit eines unvollständigen Abortes, einer Extrauteringravidität, oder unregelmässiger Blutungen z. B. bei Retroflexio usw. Auch der Palpationsbefund weist nicht eindeutig auf eine vorhergegangene Gravidität hin. Der mikroskopischen Untersuchung fällt 
deshalb die letzte Entscheidung zu. In den Fällen 1-5 ist die Diagnose der abgelaufenen Gravidität durch den Nachweis von Chorionzotten gesichert. Diese sind jedoch häufig stark degenerirt, haben nur noch Reste von Stroma und Gefässen, werden aber durch ihren Ektodermbelag kenntlich gemacht. Wie bei retinierten Resten überhaupt haben die Ektodermzellen die grösste Lebensfähigkeit. Wenn andere Gewebe schon ganz unkenntlich geworden sind, können diese Zellen noch gut färbbar sein und nur in den ersten Stadien der Degeneration sich befinden. Manche Zotte kann erst durch ihren. Belag als solche erkannt werden. Ebenso dentlich, nicht selten noch besser, sind Ektodermzellen innerhalb hyaliner Gefässwände nachweisbar. Unter allen Bestandteilen fallen diese hyalinen Gefässe mit ihren ziemlich grossen Zellen zu allererst in die Augen. Es bedarf manchmal zahlreicher Schnitte, um Chorionzotten nachzuweisen, wogegen durch die erwähnten Gefässe und Ektodermzellen von Anfang an schon unsere Aufmerksamkeit auf die Diagnose der voraufgegangenen Schwangerschaft gelenkt wird. Um möglichst sicher zu sein, haben wir dann das gesammte Ausschabungsmaterial so lange weiter geschnitten, bis andere Zeichen einer abgelaufenen Gravidität (Chorionzotten, selten Decidua) gefunden wurden. Immer wieder erwiesen sich die hyalinen Gefässe als zuverlässig. Diese Gefässe kommen nun in verschiedener Weise vor. Einen recht typischen Befund giebt die Textfig. 1, ebenso Fig. 2 u. 3 der Tafel IV. Ein Vergleich dieser Figuren mit Beschreibungen and Abbildungen einer früheren Arbeit lässt ihre Uebereinstimmung leicht erkennen. Sehr geeignet erscheint auch die Textfig. 4, die von einem puerperalen Uterus stammt, an dessen Wand noch "Reste" festsassen (Leichenpräparat). Das Endothel solcher Gefässe ist häufig, aber nicht immer, theilweise erhalten. Im Lumen liegt oft noch frisches Blat. Andere Gefässe zeigen eine auffallend breite etwas gequollene Wand (Textg. 2). Das Lumen erscheint eng, häufig aber noch mit gut erhaltenem Endothel. Endlich findet man gar nicht selten kleine obliterierte Gefässe in Gruppen nebeneinander, welche manchen Stellen ein recht zierliches Aussehen verleihen. Fast immer liegen dicht dabei andere Gefässe mit breiter hyaliner Wand und Ektodermzellen nebst erhaltenem Endothel (vgl. Taf. IV, Fig. 1). Nur selten finden sich Gefässknäuel (vgl. Langhans) inmitten von hyalinem Gewebe, umgeben von Ektodermzellen (s. Textfig.3). Nach meinen Beobachtungen fehlt in Fällen, wie den hier vorliegenden sehr häufig gut erhaltene 
88 Schickele, Mikroskopische Diagnose der abgelaufenen Schwangerschaft.

und als solche sicher zu erkennende Decidua. Aus diesem Grunde wird nur selten die Erkennung der abgelaufenen Schwangerschaft aus dem Nachweis von Decidua möglich sein. Haufen oder kleine Herde von Fibrin mit Resten von untergegangenen Zellen in Gestalt stark gefärbten, feinkörnigen Materials werden häulig beobachtet. Beweiskräftig kann dieser Befund natürlich nicht sein. "Schwangerschaftsdrüsen" ${ }^{\prime}$ sind mir recht selten begegnet (unter den angeführten Fällen sind einmal solche Drüsen erwähnt). Fast immer erscheinen

Figur 4.

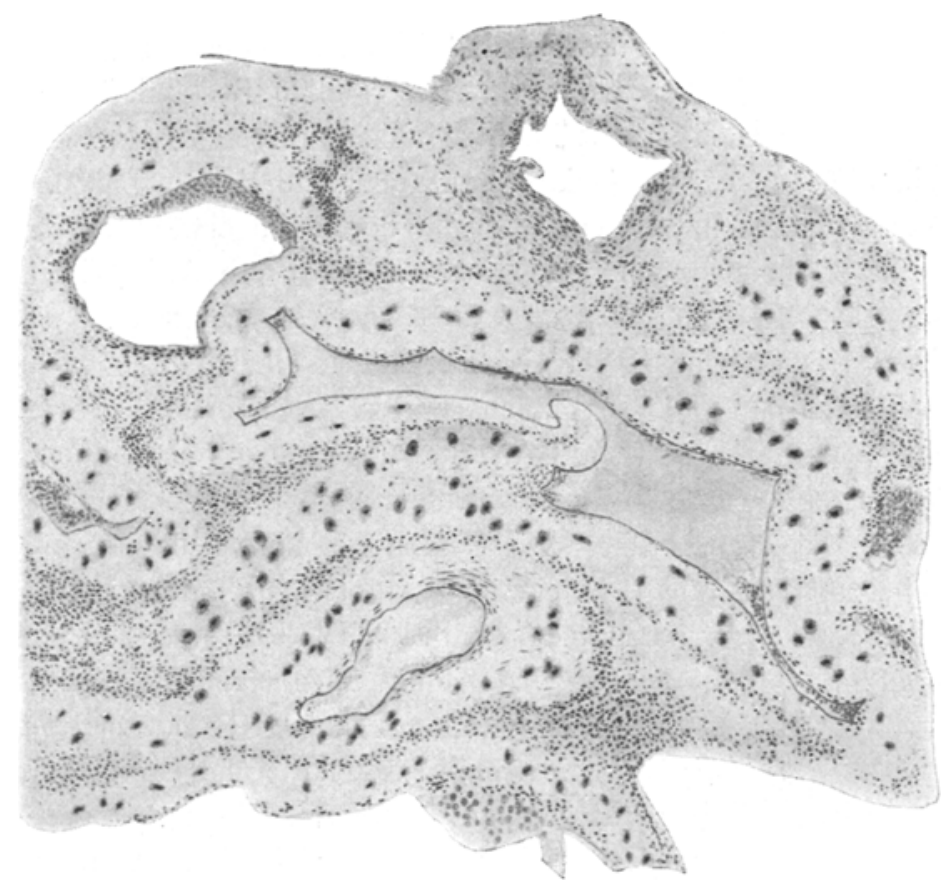

Der Uteruswand noch anhaftende retinierte Reste. (Leichenpräparat.) Chorionzotten fehlen. ZahIreiche grosse Gefässe mit meist gut erhaltenem Endothel; ihre Wand ist breit, gleichmässig hyalin; in ihr gut gefärbte Ektodermzellen.

die Drüsen normal, nicht wesentlich erweitert, mit wenig Secretion, kaum geschlängelt, jedenfalls intensiv gefärbt, die Epithelien scharf gezeichnet. Auch das Stroma der Schleimhaut ist wenig verändert. Gelegentlich trifft man etwas grösseren Zellreichthum, auch Stauung, manchmal leichte Erweiterung der Capillaren.

Hyaline Gefässe mit Ektodermzellen bedeuten unter der Voraussetzung selbstrerständlich, dass sie typisch sind ein derart sicheres Zeichen einer abgelaufenen Schwanger- 
schaft, dass ich auf ihren Nachweis allein häufig diese Diagnose stellen konnte. Nicht selten lag klinisch gar kein Grund vor, an eine Schwangerschaft zu denken. Zuweilen konnte man durch Vervollständigung der Anamnese nachträglich noch eine Andeutung finden. Bestätigt wurde die Diagnose meist durch den späteren Nachweis von Chorionzotten, wenn auch schlecht erhaltener.

Wer die eigenthümlichen Veränderungen der Arterien während der Gravidität kennt, die breiten hyalinen Streifen, in denen zahlreiche gut erhaltene Ektodermzellen liegen, gesehen hat, wird diese Verhältnisse auch in retinierten Eiresten erkennen können. Den Zusammenhang dieser Ektodermzellen mit Chorionzotten kann man allerdings nicht mehr feststellen. Letztere sind ja in den Fällen, um die es sich hier handelt, wohl immer weit zurückgebildet. Es ist nicht möglich, Angaben. über die Zeit zu machen, innerhalb deren die verschiedenen Stadien der Degeneration sich abspielen. Ausserdem glaube ich, dass diese zeitlich ausserordentlich variabel sind. Tatsache ist aber, dass fötale Ektodermzellen innerhalb der hyalinen Gefässwände am längsten erhalten bleiben. Aller Wahrscheinlichkeit nach sind sie manchmal die Jetzten und einzigen Reste einer abgelaufenen Gravidität. Wenn man diesen Gebilden eine derartige Bedeutung zuerkennen will, muss aber verlangt werden, dass sie deutlich erkennbar seien, den vorliegenden Beschreibungen und Zeichnungen vollständig entsprechend. Wenn dies der Fall, dann ist eine Verwechslung nicht möglich, da derartige Gefässveränderungen und Ektodermzellen in ähnlicher Weise ausserłalb der Gravidität nicht vorkommen. In der prämenstruellen und menstruellen Schleimhaut nimmt die Wand mancher Gefässe (besonders von Arterien) ein hyalines Aussehen an. Sie erscheint auch etwas breiter, gequollen, ihre Zellen liegen weiter auseinander. Die Zellen sind aber durchans gut erhalten und vor Allem als Bindegewebszellen nicht zu verkennen. Den Unterschied zwischen menstruellen Gefässveränderungen und den hier beschriebenen sieht man am besten an etwas grösseren Arterien, an der Grenze von Schleimhaut and Musculatur. Eine Verwechslung scheint mir unmöglich. Man kann ferner bei den beschriebenen Graviditätsveränderungen den vollständigen oder teilweisen Mangel von elastischem Gewebe nachweisen. Auch das ist ein Merkmal mehr zur sicheren Erkennung.

Die vorliegenden Gefässveränderungen mit ihren fötalen Ekto- 
90 Schickele, Mikroskopische Diagnose der abgelanfenen Schwangerschaft.

dermzellen sind schon vielen früheren Untersuchern aufgefallen (Ruge, Gebhardt, Henk, Opitz, Langhans u. A. m.). Die Beschreibung der hyalinen Beschaffenheit der Gefässwände deckt sich häufig mit der meinigen. Die in der Gefässwand befindlichen grossen Zellen sind von den meisten Untersuchern als Deciduazellen gedeutet worden. Wir können heute den Begriff der decidualen Reaction der Gefässwände wohl mit Recht aufgeben. Die grossen Zellen, welche in den hyalinen Gefässwänden der Placentarstelle liegen, sind fötale Zellen. Da diese Veränderungen der Arterien meines Wissens bei keiner anderen Gelegenheit vorkommen, dürfen wir dieser Feststellung eine besondere Bedeutung zumessen. Ich gebe diesen Befund, dem dieselbe Beweiskraft wie den Chorionzotten zukommt, zur Nachprüfung. Derjenige, der zur Kontrolle sich unbedingt auf die gleichzeitige Anwesenheit von Chorionzotten verlässt, muss darauf gefasst sein, gelegentlich das ganze Ausschabungsmaterial in Schnitte zerlegen zu müssen. Ob hyaline Gefässe mit Ektodermzellen die alleinigen Ueberbleibsel einer abgelaufenen Schwangerschaft sein können, vermag ich nicht zu sagen, da ich besonders in der letzten Zeit, von der Sicherheit dieser Befunde überzeugt, nach Zotten nicht mehr besonders gesucht habe. Es ist leicht möglich, dass alle anderen Gewebe vollständig zu Grunde gegangen oder unkenntlich sein können, dass die Gefässe aber mit ihren Ektodermzellen noch deutlich erkennbar sind.

\section{Erklärung der Abbildungen anf Tafel IV.}

Figur 1. (Ausschabung No. 249.) In dem mässig zellreichen interstitiellen Gewebe liegen mehrere Gefässe mit breiter hyaliner Wand, in ihr gut gefärbte Ektodermzellen. Das Endothel der 3 grösseren Gefässe ist gut erbalten, in dem Lumen des oberen Gefässes noch frisches Blat. In dem rechten äusseren Bezirk der Figur liegt eine Gruppe von kleinen obliterierten Gefässen, durch ihre hyaline Wand und die darin liegenden Ektodermzellen gut erkennbar.

Figuren 2 a. 3. (Ausschabung No. 206.) Zwei unweit von einander liegende Gerässe mit breiter gleichmässig hyaliner Wand, in der gut erhaltene, manchmal etwas kleine, Ektodermzellen liegen. Das Endothel dieser beiden Gefässe fehlt meistens, ist dagegen in andern wohl erbalten. Vergrösserung: Zeiss Ocul. 2. Obj. A. 
Fig.7.

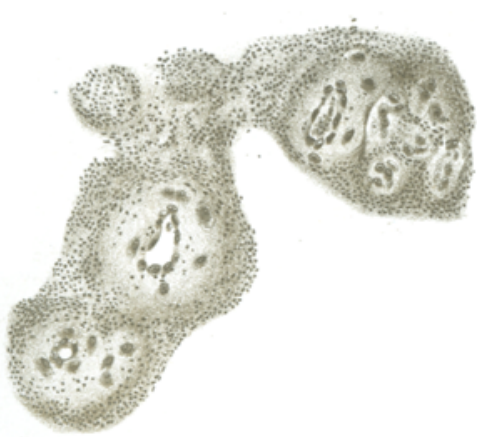

Fig. 2 .

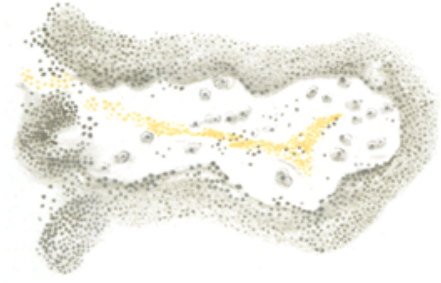

Fig.3.

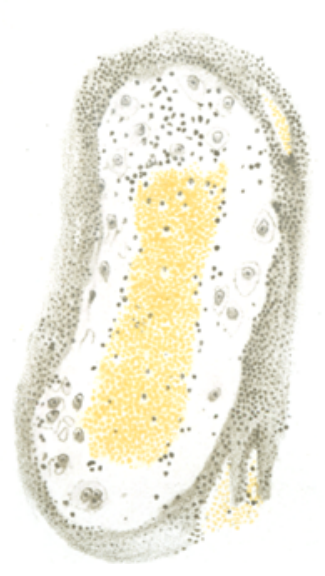

